\title{
Correlation between micro-hardness and mineral content in healthy human enamel
}

\author{
Anna Akkus ${ }^{1}$, Daniel Karasik ${ }^{2}$, Renato Roperto ${ }^{1}$ \\ ${ }^{1}$ Department of Comprehensive Care, Case Western Reserve University, Cleveland, Ohio 44106, USA \\ ${ }^{2}$ University of Chicago, Hyde Park, Illinois 60637, USA
}

Correspondence:

10900 Euclid Ave., Cleveland, OH 44106, USA

2163684351

Asp7@case.edu

\begin{abstract}
Akkus A, Karasik D, Roperto R. Correlation between micro-hardness and mineral content in healthy human enamel. J Clin Exp Dent. 2017;9(4):e56973. http://www.medicinaoral.com/odo/volumenes/v9i4/jcedv9i4p569.pdf
\end{abstract}

Received: 27/06/2016

Accepted: $18 / 07 / 2016$

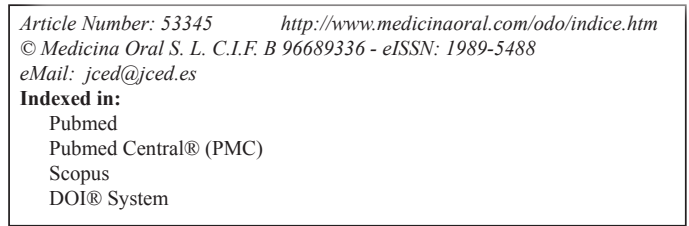

\begin{abstract}
Background: Enamel is the hardest and the stiffest tissue in the human body. The enamel undergoes multidirectional stresses, withstands multimillion chewing cycles, all while protecting the internal dentin and pulp from damage due to mechanical overload and exposure to the harsh chemical environment of the mouth. Raman spectroscopy allows to study enamel mineral content in a non-destructive and site-specific way. While Raman spectroscopy has been applied in other studies to assess tooth mineralization, there are no studies that examine the relationship between micro-hardness and mineral content of the untreated enamel. An understanding of this relationship is extremely important in a clinical context. The effect of various agents on enamel hardness was investigated, though the relationship between healthy enamel mineral content and micro-hardness remains obscure.

Material and Methods: Twenty human incisor teeth were obtained in compliance with the NIH guidelines and imaged site-specifically with a Raman microscope and evaluated with a Brinell hardness measurement device. The front portion of each tooth was divided into apical, medium and cervical regions and subsequently imaged with a Raman microscope in these three locations.

Results and Conclusions: The results demonstrated that enamel mineral content varies significantly between individuals and is correlated with the hardness of the enamel. Non-invasive, sample preparation free Raman spectroscopy was successfully employed to measure the mineral content of healthy enamel and it correlated the mineralization score to the hardness measurements of the selected cervical location. The overall level of enamel mineral content may serve as a robust predictor of patients' susceptibility to developing caries, and overall enamels wear resistance, thus allowing for the prevention of caries via clinically available methods of remineralization, fluoride treatment and frequent cleaning.
\end{abstract}

Key words: Enamel, raman spectroscopy, micro-hardness, extracted teeth.

\section{Introduction}

Enamel is the hardest and the stiffest tissue in the human body. Dental enamel is $95 \%$ mineral, $1 \%$ organic matter and $4-5 \%$ water by weight percentage (1). The enamel experiences multidirectional stresses and withstands multimillion chewing cycles, while protecting the internal dentin and pulp from damage due to mechanical overload and exposure to the harsh chemical 
environment of the mouth. Enamel mineralization is an important property that is positively correlated with the mechanical behavior of other tissues, such as bone (2) and teeth (3-5). Many dental ailments are caused by a reduction in enamel mineral content. For example, molarincisor hypo mineralization increases tooth sensitivity to food, drinks, and thermal changes, and results in restoration failure (6,7). Moreover, previous studies suggest a possible link between enamel mineral concentration and susceptibility to caries $(8,9)$.

Several authors have investigated enamel mineral content (10-14) using various characterization methods in the context of decay $(1,6)$, demineralization/remineralization processes $(15,16)$, age $(17,18)$ and disease $(19,20)$. The methods cited above, however, are limited to ex vivo laboratory conditions and are destructive to the specimens.

Raman spectroscopy offers the opportunity to study enamel mineral content in vivo (21), non-destructively and site-specifically. While Raman spectroscopy has been applied in the literature to assess tooth mineralization, there are no studies that examine the relationship between micro-hardness and mineral content of the untreated enamel. An understanding of this relationship is extremely important in a clinical context, specifically for tooth handling in vivo and structure property research inquiry of extracted teeth. It was found that enamel exposure to soft drinks adversely affects the hardness of the enamel and that this effect could be successfully counteracted via low concentration of iron supplement (22). Moreover (23) enamel from other biological species, such as sharks and crocodiles, were investigated and micro-hardness characteristics were found to be similar to that of human enamel. Although the effect of various agents on enamel hardness was effectively investigated, the relationship between enamel mineral content and micro-hardness remains obscure (24-26).

\section{Material and Methods}

\section{-Sample Preparation}

Human teeth were obtained in compliance with the National Institute of Health guidelines. The Institutional Review Board exemption was filed and approved (Protocol\#: EM-13-17). Eleven adult human incisors were extracted as part of a normal treatment plan. The teeth were collected on the date of the extraction and kept moist at all times, without any additional disinfecting treatments. A dentist assessed the enamel of the specimens selected for Raman analysis in order to ensure that healthy, intact enamel was evaluated. The samples were wrapped in wet tissue paper individually and stored in a $-20{ }^{\circ} \mathrm{C}$ freezer. Each tooth was measured with a ruler and the length of the crown was divided into 3 zones along the y axis: apical, medial and cervical, as shown in figure 1 . The cervical region was then evaluated. Prior

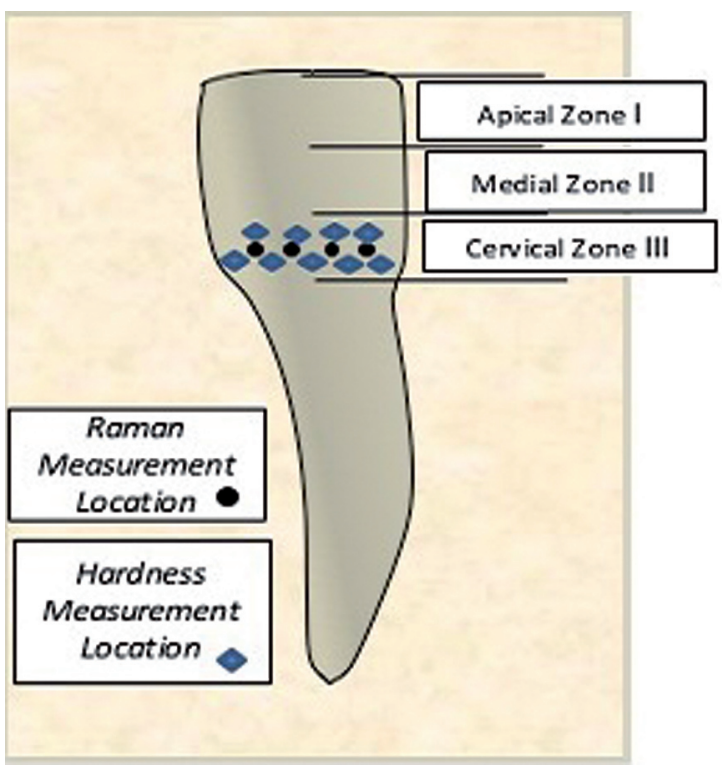

Fig. 1: Schematic of the regions included in the Raman analysis and hardness measurements.

to hardness measurements, the specimens were puttied in Whip Mix Mounding Plaster to ensure stability and horizontal, flat placement on the site of interest. Prior to the Raman analysis the specimens were thawed at room temperature for 30 minutes while being wrapped in moist tissue paper. The Raman measurements were taken in direct proximity of the indentation site from the nine hardness measurements (Fig. 1). A wet tissue paper was wrapped around the specimen to prevent dehydration during Raman scan, while exposing the region of interest for Raman analysis. Three measurements were taken within each zone in order to obtain the average mineralization within each zone.

-Raman Spectroscopy and Hardness Measurements In Raman analysis, a laser light is focused on a sample using a lens or objective. The reflected photons carry information on the type and the amount of chemical bonds. In the case of mineralized tissues, the phosphate group in the mineral of the enamel strongly scatters Raman signals. Therefore, the amplitude of the phosphate peak in the Raman spectrum is proportional to the amount of mineral content. It is for this reason that Raman spectroscopy has long been a powerful tool for assessing mineral content, not only in the dentistry, but also other mineralized tissue, such as bone. In the current study, an 10x objective was used to focus the laser light. The resulting excitation spot was about $10 \mu \mathrm{m}$ in diameter and the penetration of the laser was within $100 \mu \mathrm{m}$.

A Raman microscope (Labram Xplora, Horiba Jobin Yvon, Edison, NJ) with a laser source at $785 \mathrm{~nm}$ was used. Measurements were performed using a 1200 lines/mm grating, which provided a wavenumber resolution of 1.25 pixels $/ \mathrm{cm}^{-1}$. Six acquisitions per point were taken. The Raman wavenumber shift measured by the 
system was calibrated using the known $520.7 \mathrm{~cm}^{-1}$ peak of a $\mathrm{Si}$ wafer. The mineralization was assessed based on the intensity of the $960 \mathrm{~cm}^{-1}$ peak of the phosphate (27) symmetric stretch band (1).

The specimens were positioned in Brinell hardness measurement device. Nine locations were measured under: $500 \mathrm{~g}$ load was applied for 15 seconds (Fig. 1).

\section{Results}

The height of the $960 \mathrm{~cm}^{-1}$ phosphate peak was measured to compare the mineral content levels in 20 incisors in the cervical area. The highest Raman-based mineralization intensity was about five times greater than the lowest measured mineral content (Fig. 2). The average value of enamel mineral content in the cervical region of the tooth was obtained by pooling together the measurements of the corresponding zone of 20 individuals with an average number of four measurements per tooth specimen. When the measurements were pooled within the cervical region, incisor hardness varied dramatically depending on the region (Fig. 3). Moreover a correlation between the amount of mineral and hardness was observed. The high hardness values corresponded to the high mineral content measurements obtained with the Raman microscope.

\section{Discussion}

The results demonstrated that mineralization varies substantially between individuals and is correlated with the hardness of the enamel. In a previous study, it was also observed that the cervical enamel has the least mineralization with a populational average (28). The lower mineralization scores for the cervical region may be associated with a hygiene index. Dental plaque can normally be found on the cervical area of the tooth. Due to the highly acidic environment of the mouth, dental plaque can easily deactivate the enamel buffering capacity. Thus, it is important to understand the relationship

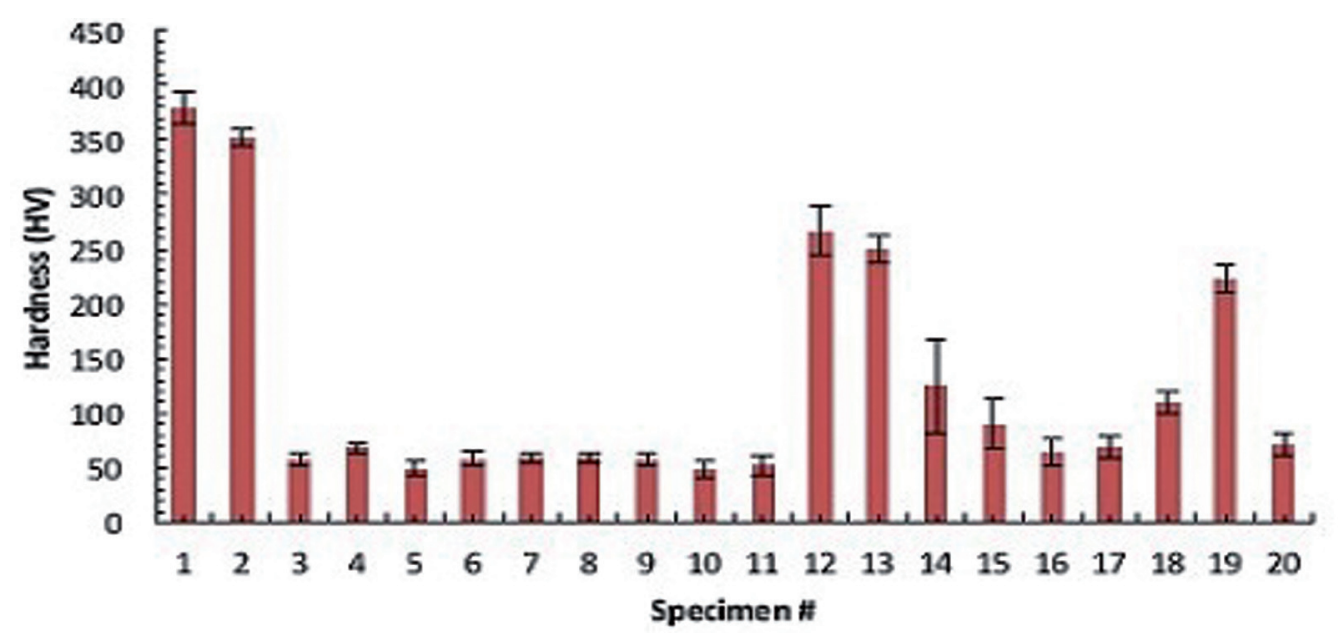

Fig. 2: Hardness measurements for twenty human incisors in the cervical region of the crown.

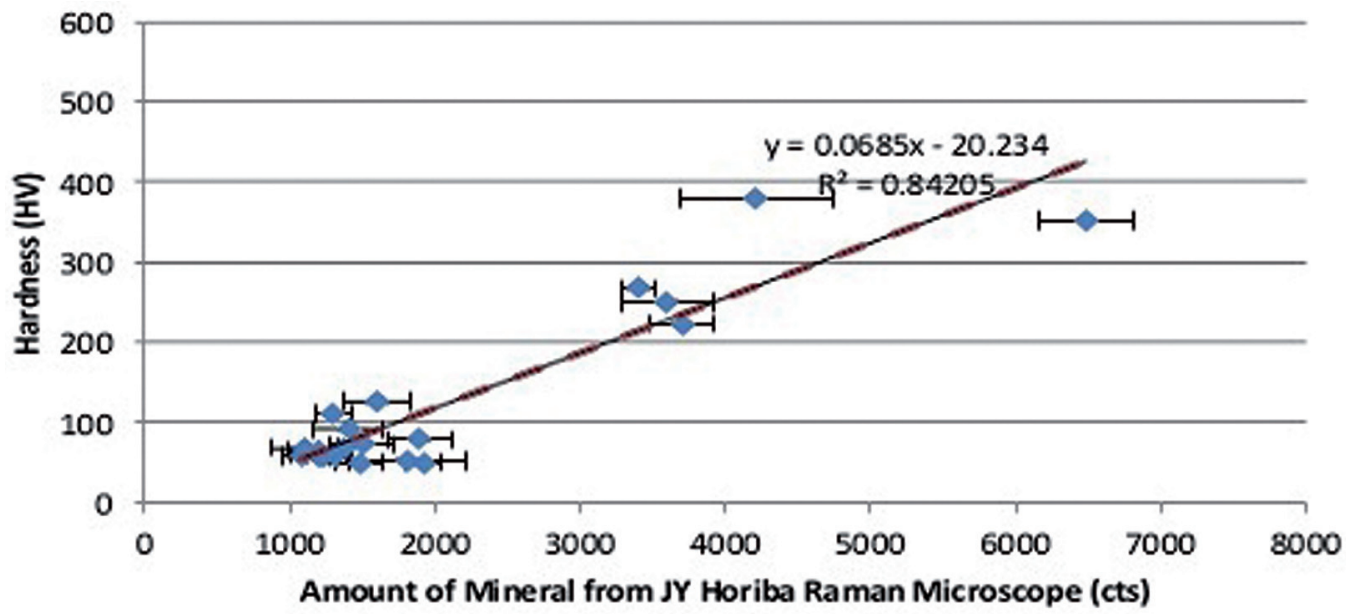

Fig. 3: Correlation between hardness and mineral content in human enamel. 
between basic material properties and differences in degrees of mineralization in the cervical region of the tooth.

A range of values of mineral concentration has been reported (29-31) in previous studies (EDS, XRD analysis, X-ray microtomography). The methods employed, however, require destruction of the specimens, such as sectioning of the tooth into slices. Therefore, they are not clinically applicable. While computed tomography is viable, the spatial resolution of clinically available cone-beam systems is insufficient to capture the enamel layer. Subjection of the patient to ionizing radiation is another limitation. The Raman spectroscopy based assessment of mineralization holds a substantial promise for clinical application since Raman spectroscopy is noninvasive and does not require sample preparation. It is particularly informative in evaluating the mineral content of enamel due to the observed correlation between enamel hardness and overall measured mineral content in Raman microscope.

It has been suggested that the lower mineral concentration may be translated into increased porosity and is possibly linked to higher caries susceptibility. Moreover the results of this study demonstrate the lower hardness values for the enamel with lower mineral content score (32). Early identification of individuals with lower overall enamel mineralization may be a valuable way to begin to intervene before the development of caries.

\section{Conclusions}

Non-invasive, sample-preparation free Raman spectroscopy was successfully employed to measure mineral content of healthy enamel and the measurements were used to find a correlation of the mineralization score to the hardness measurements of the selected cervical location. It was demonstrated that cervical region of the healthy enamel in the pool of the incisor specimens exhibit the lowest mineralization content in the cervical region of the crown. The overall level of enamel mineral content may serve as a robust predictor of patients' susceptibility to developing caries, and overall enamels wear resistance, thus granting the opportunity to prevent the caries via clinically available methods of remineralization, fluoride treatment and frequent cleaning.

\section{References}

1. Baldassarri M1, Margolis HC, Beniash E. Compositional determinants of mechanical properties of enamel. J Dent Res. 2008;87:645-9. 2. Leichter I, Margulies JY, Weinreb A, Mizrahi J, Robin GC, Conforty $\mathrm{B}$, et al. The relationship between bone density, mineral content, and mechanical strength in the femoral neck. Clin Orthop Relat Res. 1982;163:272-81.

3. Lopes FC, Roperto R, Akkus A, Akkus O, Souza-Gabriel AE, Sousa-Neto MD. Effects of different lasers on organic/inorganic ratio of radicular dentin. Lasers Med Sci. 2016;31:415-20.

4. Kinney JH, Balooch M, Marshall SJ, Marshall GW Jr, Weihs TP. Hardness and Young's modulus of human peritubular and intertubular dentine. Arch Oral Biol. 1996;41:9-13.
5. Kodaka T, Debari K, Yamada M, Kuroiwa M. Correlation between microhardness and mineral content in sound human enamel (short communication). Caries Res.1992;26:139-41.

6. William V, Burrow MF, Palamara JE, Messer LB. Microshear bond strength of resin composite to teeth affected by molar hypomineralization using 2 adhesive systems. Pediatr Dent. 2006;28:233-41.

7. Yang S, Li B, Akkus A, Akkus O, Lang L. Wide-field Raman imaging of dental lesions. Analyst. 2014;139:3107-14.

8. Shellis RP. Relationship between human enamel structure and the formation of caries-like lesions in vitro. Arch Oral Biol. 1984;29:97581.

9. Targino AG, Rosenblatt A, Oliveira AF, Chaves AM, Santos VE. The relationship of enamel defects and caries: a cohort study. Oral Dis. 2011;17:420-6.

10. He B, Huang S, Zhang C, Jing J, Hao Y, Xiao L, et al. Mineral densities and elemental content in different layers of healthy human enamel with varying teeth age. Arch Oral Biol. 2011;56:997-1004.

11. Wong FS, Elliott JC, Davis GR, Anderson P. X-ray microtomographic study of mineral distribution in enamel of mandibular rat incisors. J Anat. 2000;196:405-13.

12. Wong FS, Anderson P, Fan H, Davis GR. X-ray microtomographic study of mineral concentration distribution in deciduous enamel. Arch Oral Biol. 2004;49:937-44.

13. Farah RA, Swain MV, Drummond BK, Cook R, Atieh M. Mineral density of hypomineralised enamel. J Dent. 2010;38:50-8.

14. Jeng YR, Lin TT, Wong TY, Chang HJ, Shieh DB. Nano-mechanical properties of fluoride-treated enamel surfaces. J Dent Res. 2008;87:381-5.

15. Efeoglu N, Wood D, Efeoglu C. Microcomputerised tomography evaluation of $10 \%$ carbamide peroxide applied to enamel. J Dent. 2005;33:561-7.

16. Efeoglu N, Wood DJ, Efeoglu C. Thirty-five percent carbamide peroxide application causes in vitro demineralization of enamel. Dent Mater. 2007;23:900-4

17. He B, Huang S, Jing J, Hao Y. Measurement of hydroxyapatite density and Knoop hardness in sound human enamel and a correlational analysis between them. Arch Oral Biol. 2010;55:134-41.

18. Zheng Q, Xu H, Song F, Zhang L, Zhou X, Shao Y, Huang D. Spatial distribution of the human enamel fracture toughness with aging. $J$ Mech Behav Biomed Mater. 2013;26:148-54.

19. Atar M, Davis GR, Verry P, Wong FS. Enamel mineral concentration in diabetic rodents. Eur Arch Paediatr Dent. 2007;8:195-200.

20. Koehne T, Marshall RP, Jeschke A, Kahl-Nieke B, Schinke T, Amling M. Osteopetrosis, osteopetrorickets and hypophosphatemic rickets differentially affect dentin and enamel mineralization. Bone. 2013;53:25-33.

21. Ko AC, Hewko M, Sowa MG, Dong CC, Cleghorn B, ChooSmith LP. Early dental caries detection using a fibre-optic coupled polarization-resolved Raman spectroscopic system. Opt Express. 2008:16:6274-84.

22. Xavier AM, Rai K, Hegde AM, Shetty S. A spectroscopic and surface microhardness study on enamel exposed to beverages supplemented with lower iron concentrations. J Clin Pediatr Dent. 2015;39:161-7.

23. Enax J, Prymak O, Raabe D, Epple M. Structure, composition, and mechanical properties of shark teeth. J Struct Biol. 2012;178:290-9.

24. Leandro GA, Attia ML, Cavalli V, do Rego MA, Liporoni PC. Effects of $10 \%$ carbamide peroxide treatment and sodium fluoride therapies on human enamel surface microhardness. Gen Dent. 2008;56:274-7.

25. Majeed A, Grobler SR, Moola MH, Rossouw RJ, van Kotze TJ. Effect of four different opalescence tooth-whitening products on enamel microhardness. SADJ. 2008;63:282-4,286.

26. Attin T, Schmidlin PR, Wegehaupt F, Wiegand A. Influence of study design on the impact of bleaching agents on dental enamel microhardness: a review. Dent Mater. 2009;25:143-57.

27. Leung Y, Morris MD. Characterization of the chemical interactions betwen 4-MET and enamel by Raman spectroscopy. Dent Mater. 1995; 11:191-5. 
28. Akkus A, Akkus O, Roperto R, Lang L. Investigation of Intraand Inter-individual Variations of Mineralisation in Healthy Permanent Human Enamel by Raman Spectroscopy. Oral Health Prev Dent. 2016;14:321-7.

29. STACK MV. Organic constituents of enamel. J Am Dent Assoc. 1954;48:297-306.

30. Weatherell JA, Weidmann SM, Hamm SM. Sampling of enamel particles by means of strong acids for density measurements. Arch Oral Biol. 1966;11:107-12.

31. Weatherell JA, Weidmann SM, Hamm SM. Density patterns in enamel. Caries Res. 1967;1:42-51.

32. Yeni YN, Yerramshetty J, Akkus O, Pechey C, Les CM. Effect of fixation and embedding on Raman spectroscopic analysis of bone tissue. Calcif Tissue Int. 2006;78:363-71.

\section{Conflict of Interest}

The authors have declared that no conflict of interest exist. 